CVIA

CASE REPORT

pISSN 2508-707X / elSSN 2508-7088 https://doi.org/10.22468/cvia.2018.00255 CVIA 2019;3(1):19-23

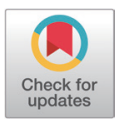

\section{Left Ventricular Noncompaction Associated with Hypertrophic Cardiomyopathy: Morphologic and Functional Evaluation with Multidetector CT}

\author{
Heon Lee ${ }^{1}$, Jae-Wook Lee ${ }^{1}$, Felix G. Meine ${ }^{2,3}$, U. Joseph Schoepf $f^{2,4}$ \\ 'Department of Radiology, Soonchunhyang University Bucheon Hospital, Bucheon, Korea \\ 2Department of Radiology and Radiological Science, Medical University of South Carolina, \\ Charleston, SC, USA \\ ${ }^{3}$ Institute for Diagnostic and Interventional Radiology, University of Rostock, Germany \\ ${ }^{4}$ Division of Cardiology, Department of Medicine, Medical University of South Carolina, \\ Charleston, SC, USA
}

Received: October 30, 2018

Revised: December 14, 2018

Accepted: December 24, 2018

Corresponding author

U. Joseph Schoepf, MD

Department of Radiology and

Radiological Science,

Medical University of South Carolina,

Ashley River Tower, MSC 226,

25 Courtenay Drive, Charleston,

SC 29401, USA

Tel: 1-843-876-7146

Fax: 1-843-876-3157

E-mail: schoepf@musc.edu

\begin{abstract}
Hypertrophic cardiomyopathy and left ventricular (LV) noncompaction are American Heart Association-recognized cardiomyopathies with distinct clinical and pathoanatomical features that are presumed to have different etiologies. We present two cases with features of both hypertrophic cardiomyopathy and LV noncompaction, with emphasis on the emerging role of cardiac CT as a single imaging modality for morphological and functional evaluation of the heart.
\end{abstract}
Key words Isolated noncompaction of the ventricular myocardium . Hypertrophic cardiomyopathy · Multidector-row computed tomography Genetics.

\section{INTRODUCTION}

We describe two patients with features of both hypertrophic obstructive cardiomyopathy (HCM) and left ventricular (LV) noncompaction. HCM and LV noncompaction are American Heart Association-recognized cardiomyopathies with distinct clinical and pathoanatomical features [1,2]. Our cases show that both conditions can occur simultaneously and thus support the previously published hypothesis that these two disease entities result from the same genetic mutations [3,4]. With these cases showing a combination of two rare cardiac diseases, we emphasize the utility of cardiac CT for the anatomical assessment of coronary arteries and the evaluation of ventricular morphology and function.

(c) This is an Open Access article distributed under the terms of the Creative Commons Attribution Non-Commercial License (https://creativecommons.org/licenses/bync/4.0) which permits unrestricted non-commercial use, distribution, and reproduction in any medium, provided the original work is properly cited.

\section{CASE REPORT}

\section{Case 1}

A 75-year-old female was referred for cardiac CT after echocardiography had demonstrated asymmetrical myocardial hypertrophy. Cardiac CT with retrospective ECG gating showed no evidence of coronary artery disease (CAD) (Fig. 1A) and revealed asymmetric septal hypertrophy, systolic anterior motion of the thickened anteromedial mitral leaflet (Fig. 1B, arrow), and a dynamic LV outflow tract (LVOT) obstruction consistent with HCM (Fig. 1B, asterisk). In addition, prominent trabeculation was noted in the lateral and apical wall of the LV (Fig. 1C). The maximum ratio between noncompacted (NC) to compacted (C) myocardium in end-diastole exceeded 2.3:1, suggesting LV noncompaction. The diagnosis of HCM in this patient was also supported by echocardiographic criteria, including a septal/posterior wall thickness ratio $>1.6$ (Fig. 1D) and a resting LVOT obstruction with a peak pressure gradient of $38 \mathrm{~mm} \mathrm{Hg}$ (Fig. 1E). Since the clinical and imaging evalua- 

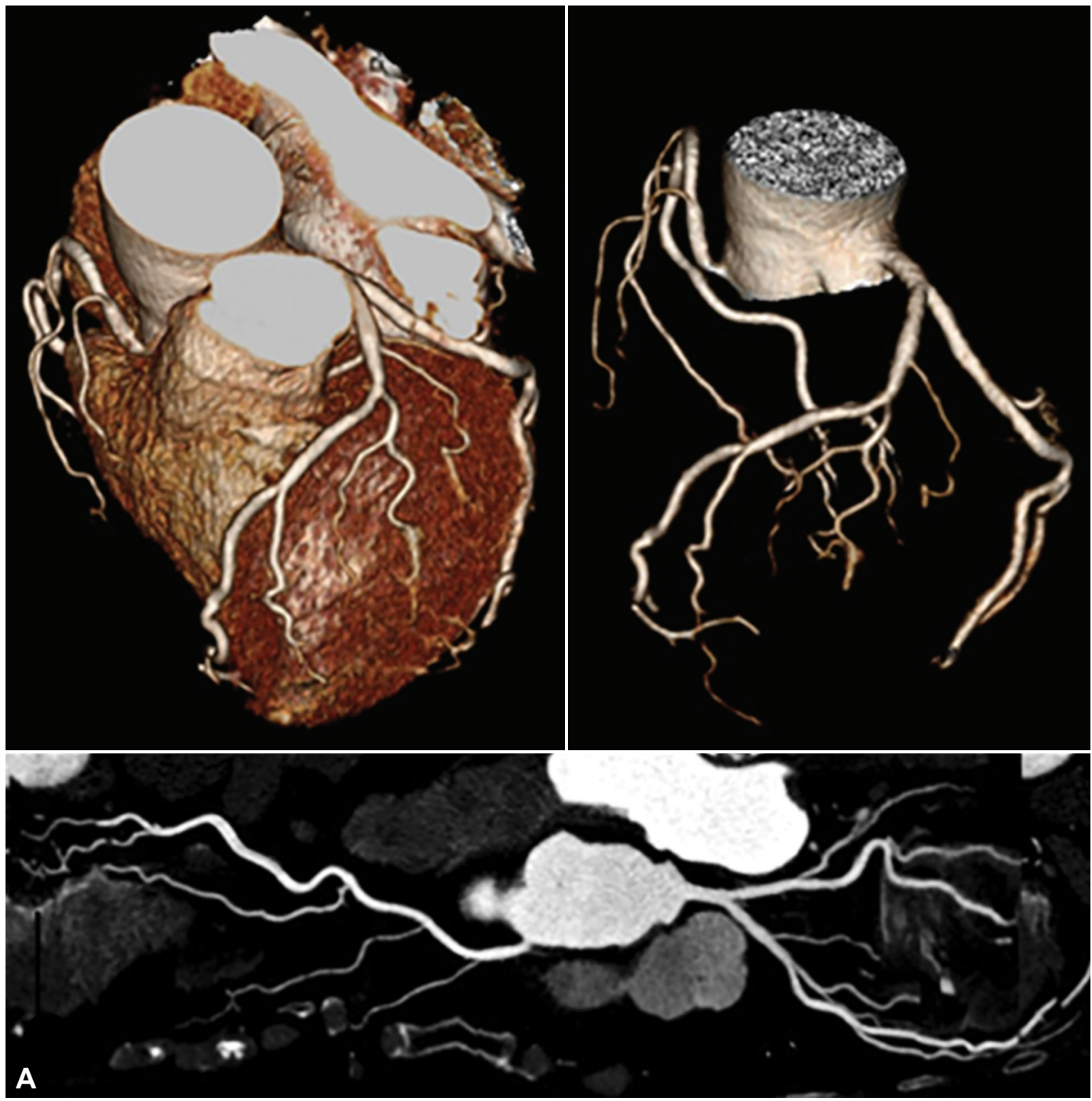

Fig. 1. Imaging for Case 1. Cardiac CT shows normal coronary arteries (A) and revealed asymmetric septal hypertrophy (B, *), systolic anterior motion of the thickened anteromedial mitral leaflet and a dynamic LVOT obstruction (B, arrow). In addition, prominent trabeculation was noted in the apicolateral wall of the left ventricle (C). Echocardiography demonstrated a septal/posterior wall thickness ratio $>1.6$ (D) and a resting LVOT obstruction with a peak pressure gradient of $38 \mathrm{~mm} \mathrm{Hg}(\mathrm{E})$. LVOT: left ventricular outflow tract.

tion supported the diagnosis of LV noncompaction and HCM associated with LVOT and without CAD, the patient received supportive treatment for aortic stenosis and heart failure and experienced symptom improvement.

\section{Case 2}

A 59-year-old man with a history of atrial fibrillation was admitted with vague chest pain for several days prior to admission. He was referred for cardiac CT after echocardiography demonstrated asymmetrical septal hypertrophy. Ejection fraction was measured at $62 \%$ by the echocardiogram. Cardiac CT with retrospective ECG gating depicted small calcified plaques without significant luminal stenosis in the proximal segment of the left anterior descending artery and its first diagonal branch (Fig. 2A). Cardiac CT also demonstrated asymmetrical septal hypertro- phy of the LVOT and prominent trabeculation noted in the lateral and apical wall of the LV (Fig. 2B). The maximum ratio between $\mathrm{NC}$ to $\mathrm{C}$ myocardium in end-diastole was more than 2.5:1, suggesting LV noncompaction. Since image evaluation supported the combination of LV noncompaction and HCM without significant $\mathrm{CAD}$, the patient received supportive treatment and was discharged with improvement in his symptoms.

\section{DISCUSSION}

Our cases support the hypothesis that HCM and features of LV noncompaction can appear simultaneously and may result from the same genetic mutation.

HCM is a condition characterized by a diffuse or segmental thickening of LV myocardium with a non-dilated and hyperdy- 

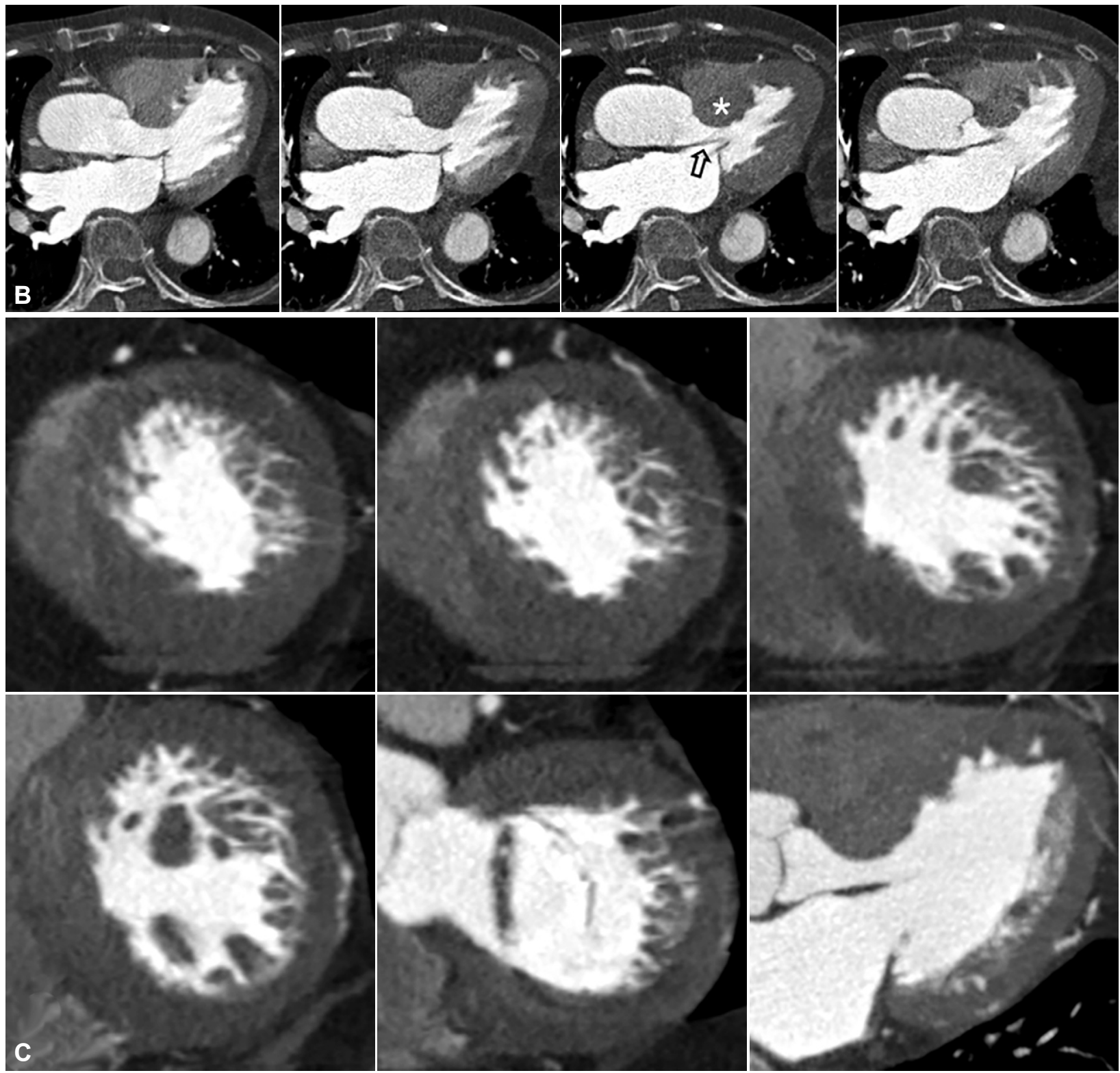

C
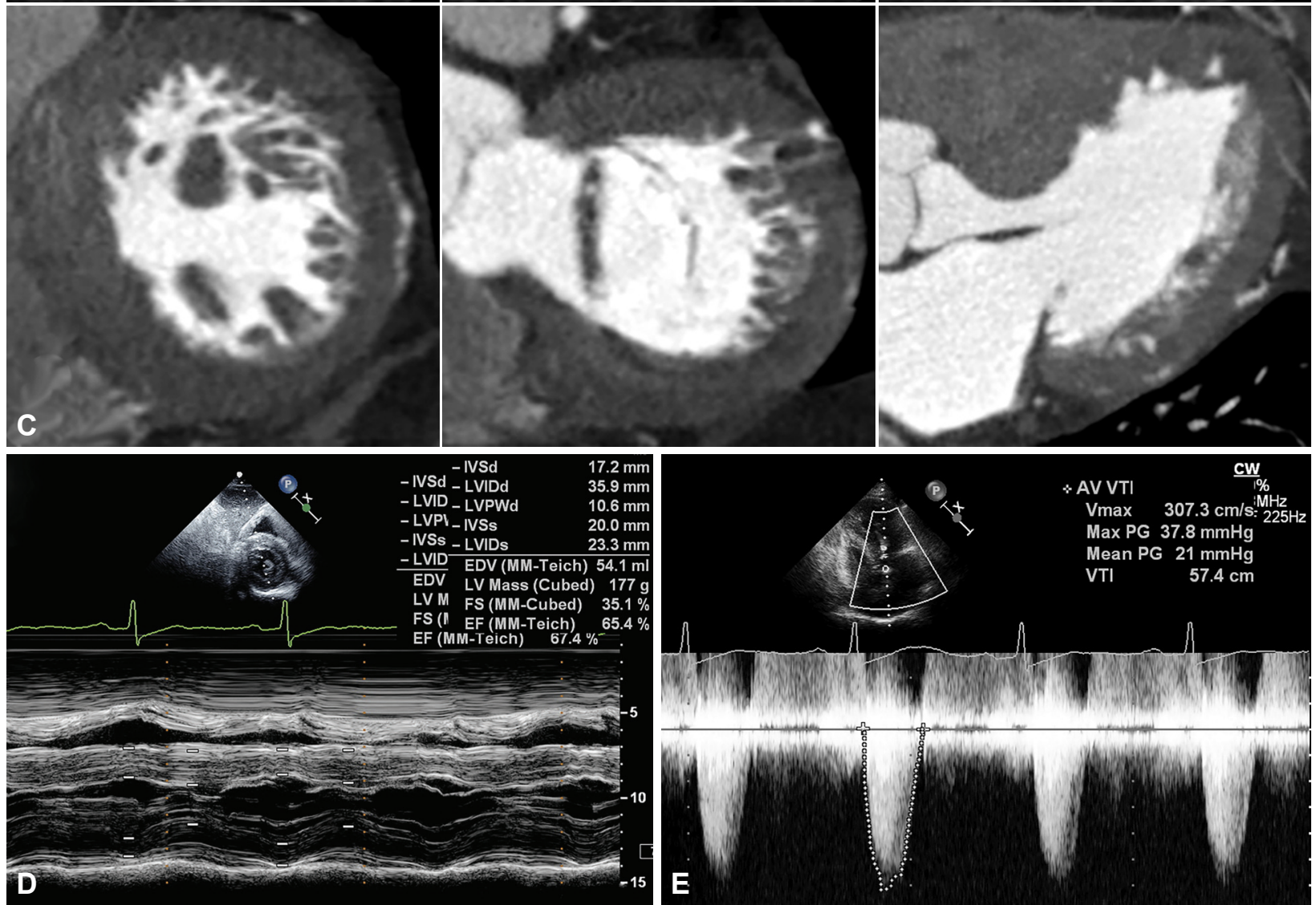

Fig. 1. Imaging for Case 1. Cardiac CT shows normal coronary arteries (A) and revealed asymmetric septal hypertrophy $\left(B,{ }^{*}\right)$, systolic anterior motion of the thickened anteromedial mitral leaflet and a dynamic LVOT obstruction ( $\mathrm{B}$, arrow). In addition, prominent trabeculation was noted in the apicolateral wall of the left ventricle (C). Echocardiography demonstrated a septal/posterior wall thickness ratio $>1.6$ (D) and a resting LVOT obstruction with a peak pressure gradient of $38 \mathrm{~mm} \mathrm{Hg}(\mathrm{E})$. LVOT: left ventricular outflow tract. 

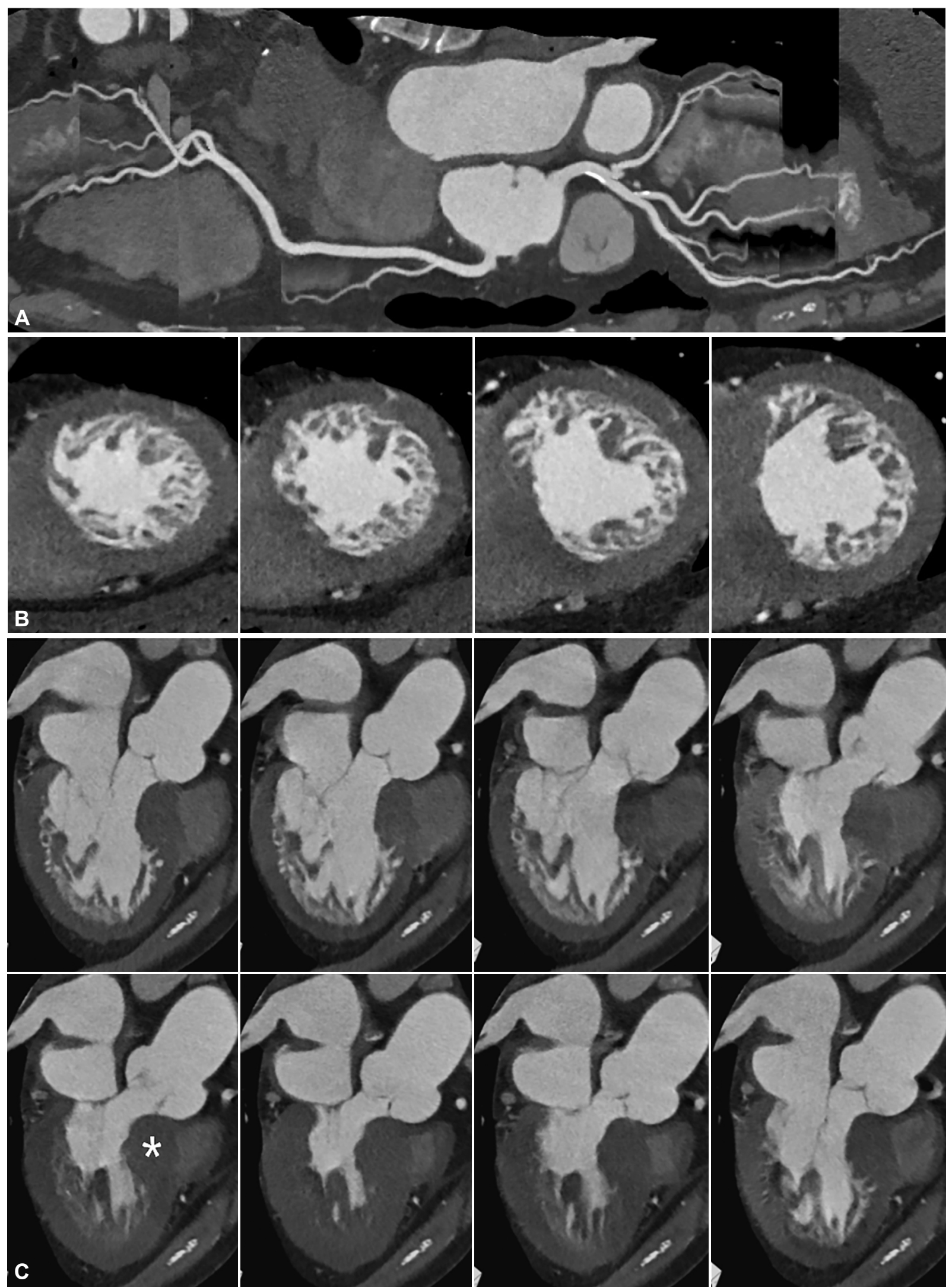

Fig. 2. Imaging for Case 2. Cardiac CT shows small calcified plaques without luminal stenosis on the left anterior descending artery and diagonal branch (A) and revealed prominent trabeculation in the apicolateral wall of the left ventricle (B) with a 2.5:1 ratio of noncompacted to compacted myocardium. Asymmetric septal hypertrophy $\left(\mathrm{C},{ }^{*}\right)$ is noted without systolic anterior wall motion of the mitral leaflet. 
namic chamber and no other cardiac or systemic disease [1,3]. HCM is one of the most common genetic cardiac diseases, with a reported prevalence of around $0.2 \%$. The imaging diagnosis of HCM is based on a maximal LV wall thickness $\geq 15$ $\mathrm{mm}$ in the end-diastolic phase [1,5]. HCM is associated with a wide variety of phenotypes and a diverse clinical course, including sudden cardiac death in young adults [1,3]. HCM follows as a Mendelian autosomal dominant inheritance pattern and is caused by a missense mutation in one of the sarcomeric genes that encode cardiac sarcomeric protein $[1,2,4]$. The pathologic hallmark of HCM is myocyte disorganization/disarray, which is widespread throughout the $\mathrm{LV}$ and, to a lesser extent, in the right ventricle.

LV noncompaction is a rare cardiomyopathy, with a prevalence of less than $0.02 \%$, characterized by excessive trabeculations of the $\mathrm{LV}$ with two distinct layers of $\mathrm{NC}$ and $\mathrm{C}$ myocardium. It is diagnosed and also differentiated from hypertrabeculation by a ratio of NC/C myocardium $>2.0$ at end-systole on echocardiography, and $>2.3$ at end-diastole on MR [6]. The natural history of LV noncompaction is unclear. Patients may be asymptomatic or suffer from dyspnea, arrhythmia, and systolic heart failure, thromboembolic events secondary to atrial fibrillation, or sudden cardiac death. Although not much is known about its inheritance pattern, LV noncompaction is a genetically heterogeneous entity with a sporadic and familial form [2].

Recently, different mutations in sarcomeric genes, which previously have been found to be involved in the pathogenesis of HCM, have been also identified in patients with isolated LV noncompaction without myocardial hypertrophy. Thus, there seems to be a shared genetic origin of these two different cardiomyopathic phenotypes [1-4,6]. Although genetic counseling may serve these patients and their families, it was not performed in our patients due to practical reasons. At present, the diagnosis of HCM and noncompaction as a clinical entity is still based on an imaging modality that directly visualizes myocardial hypertrophy and trabeculation, rendering genetic study of little benefit compared to the cost in the management of disease [1-3,6].

The frequency of cardiac CT as an emerging modality for si- multaneous evaluation of cardiac morphology and function, as well as CAD, has increased. Although echocardiography remains the modality of choice for diagnosing HCM and LV noncompaction, in our cases, cardiac CT enabled multi-planar reconstructions in any plane desired and offered comprehensive assessment of general cardiac morphology in addition to providing high-quality angiographic images of the coronary tree $[1,5]$. In both cases, cardiac CT scans were performed to exclude $\mathrm{CAD}$ and subsequently provided morphological and functional information about the myocardium, cardiac chambers, and valve motion. Collectively, this analysis allowed for the diagnosis of both HCM and noncompaction in a single patient.

In summary, our cases suggest that these cardiomyopathies may have a similar genetic etiology, and that the phenotypic expression of both diseases can occur in the same patient. These cases also highlight the integrative nature of cardiac CT as a single, comprehensive imaging tool for simultaneous morphological and functional evaluation of the heart.

\section{Conflicts of Interest}

Dr. Schoepf is a consultant for and receives research support from Bayer, Bracco, Elucid BioImaging, GE, Guerbet, HeartFlow, and Siemens. The other authors have no conflict of interest to disclose.

\section{REFERENCES}

1. Chun EJ, Choi SI, Jin KN, Kwag HJ, Kim YJ, Choi BW, et al. Hypertrophic cardiomyopathy: assessment with MR imaging and multidetector CT. Radiographics 2010;30:1309-1328.

2. Oechslin E, Jenni R. Left ventricular non-compaction revisited: a distinct phenotype with genetic heterogeneity? Eur Heart J 2011;32:1446-1456.

3. Kelley-Hedgepeth A, Towbin JA, Maron MS. Images in cardiovascular medicine. Overlapping phenotypes: left ventricular noncompaction and hypertrophic cardiomyopathy. Circulation 2009;119:588-589.

4. Ramineni R, Merla R, Chernobelsky A. Noncompaction of ventricular myocardium associated with hypertrophic cardiomyopathy and polycystic kidney disease. Am J Med Sci 2010;339:383-386.

5. Kim AM, Hunter TA, McQuillan BF, Franco DF, Griffith TP, Carter BW, et al. Imaging in congenital and hereditary abnormalities of the interventricular septum: clinical anatomy and diagnostic clues. J Thorac Imaging 2018;33:147-155.

6. Arbustini E, Weidemann F, Hall JL. Left ventricular noncompaction: a distinct cardiomyopathy or a trait shared by different cardiac diseases? J Am Coll Cardiol 2014;64:1840-1850. 